\title{
Transport Layer Multipath on Wireless Sensor Network Backhaul Links
}

\author{
Nikolas Stephan \\ Dept. of Computer Science \\ University College London \\ n.stephan@cs.ucl.ac.uk
}

\author{
Socrates Varakliotis \\ Dept. of Computer Science \\ University College London \\ s.varakliotis@cs.ucl.ac.uk
}

\author{
Peter T. Kirstein \\ Dept. of Computer Science \\ University College London \\ p.kirstein@cs.ucl.ac.uk
}

\begin{abstract}
With the increasing popularity of wireless sensor networks in hostile environments it would be desirable to have more reliable ways of delivering collected information to its destination. With a common scenario involving nodes in a network relaying data back to some kind of router, which is connected to a backhaul network the question arises whether this single point of failure could not be improved, especially in those cases where mobile networks are involved (e.g. PAN or VAN).

This paper demonstrates how this single point of failure can be improved upon in regards to network failures, if not hardware failures which must be tackled independently. The suggested solution consists of bundling multiple connections at the transport layer to improve the reliability by employing redundancy. It also has a number of further advantages such as helping to reduce the delay in mobility scenarios by cutting back on handover delays and offering alternate paths for retransmissions.

In the past multipath research in wireless sensor networks has largely focused on the data link, or network layer [2]. We hope to show that there are also reasons to employ it at the transport layer in certain situations.
\end{abstract}

\section{Keywords}

multipath, redundancy, transport layer multi-homing

\section{INTRODUCTION}

Though often ignored due to a perception of inelegance, multihoming at the transport layer can offer significant advantages over solutions at lower layers. Load balancing can usually be performed more effectively as there is more information on the actual throughput and current health of each utilised connection. In addition to this it usually makes it significantly easier to load balance between two connections on a per packet basis, rather than simply switching to a second connection in case of a failure on the primary.

Permission to make digital or hard copies of all or part of this work for personal or classroom use is granted without fee provided that copies are not made or distributed for profit or commercial advantage and that copies bear this notice and the full citation on the first page. To copy otherwise, to republish, to post on servers or to redistribute to lists, requires prior specific permission and/or a fee.

Copyright 200X ACM X-XXXXX-XX-X/XX/XX ...\$5.00.
Although applicable to all types of networks and perhaps of particular interest to the Internet, this paper will focus on the use of multipath transport protocols for the transmission of data collected from sensor networks with a specific view towards emergency situations. There are many very diverse areas in which these protocols offer advantages and some functionality spans all these. However, to discuss some of the more nuanced advantages a specific subject area is advisable.

\section{BACKGROUND}

The general concept of transport layer multipath is to allow a standard socket to be made up of a number of distinct end-to-end paths without requiring any changes at other layers and most importantly in applications themselves.

A simple outline of how this could be architected is shown in Figure 1. In this case the application creates a socket in accordance with the sockets API (which is not an actual layer but simply a specification of interfaces). The difference is that a layer has been added in the form of the logical socket which the application interfaces directly with. This logical socket then intelligently balances outbound data between the actual sockets below it, as well as merging incoming data from all sockets into a single stream. In a normal case the application would talk directly to actual sockets in the bottom layer.

If correctly approached the resulting implementation of the protocol will be entirely backwards compatible and opaque to applications so that it seems as if they are transmitting data through a standard socket.

In addition to this one must likely add some additional information to successfully establish connections. On the level of individual packets this includes things such as a connection identifier to allow mapping sockets to logical sockets and possibly sequence numbers, depending on the protocol in question and design of the multipath implementation built on it. Furthermore there must be a facility to transmit addresses between the hosts so that sockets beside the initial one can be connected. The complexity of this greatly depends on the protocol being used, as some protocols, such as SCTP, already have facilities for this exchange.

\section{RELATED WORK}

There are a number of implementations of transport layer multipath protocols. Generally research in this area has largely focused on specific aspects of the implementation such as load balancing, mobility or reliability rather than applications that may benefit from such an implementation. 


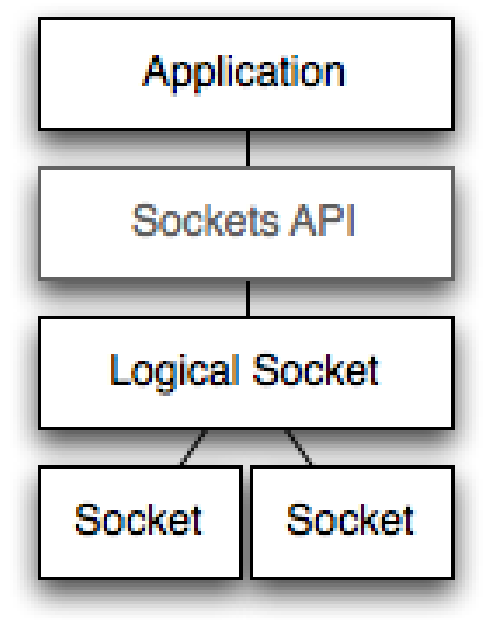

Figure 1: A diagram showing the different layers in transport layer multipath.

It is also important to note that implementing this at the transport layer does not make equal sense for all protocols. UDP is one example where an implementation at the network layer, such as SHIM6 [8] would most likely yield similar results while being more general. A certain amount of thought should therefore be given to where such an implementation makes sense at the transport layer and where a more general solution would be advisable.

One of the authors recently worked on an implementation of multipath for the Linux kernel TCP stack [3] and work on a userspace implementation by another group is ongoing. The results so far show that a reliable implementation is possible, works as expected to load balance reliably between the two connections and offers functional failover redundancy.

In addition to this there is a lot of work covering parts of the proposed functionality. One example of this is an attempt to implement a mobile TCP socket [7].

There has also been some previous work to create an implementation of multipath for the Stream Control Transmission Protocol [1][4]. This is slightly different in that SCTP already employs a model in which connections can be bundled to allow for failover redundancy and the required additions therefore mainly centre around being able to load balance between these multiple connections and use them concurrently to raise overall throughput.

\section{FEATURES}

\subsection{Redundancy}

One of the advantages of this solution is that it is not simply failover redundancy in that the switch does not occur after a failure but spreads the load between multiple connections throughout the sockets entire lifespan. This means in the case of a failure the switch to another connection is instant upon detection and simply consists of no longer sending through that path. The limiting factors in this case are simply how long it takes to detect failures and how aggressive one is in assuming that they are permanent.

\subsection{Load-Balancing}

The option of load balancing between different paths is where transport layer multipath has some significant advantages over solutions at lower layers. The fact that, depending on the protocol in question, one has access to many transmission metrics allows the load balancing to be done based on advanced metrics such as throughput, delay, packet loss and many others. How these are used mainly depends on what requirements one has and what environment the system is to be used in.

In addition to this one could also introduce external artificial metrics such as blocking a path so it is only used when there is no other option. This could help in avoiding overloading a wireless connection for example. This means one can have the reliability of an instant backup connection without actually using when the main connection is functioning reliably.

\subsection{Throughput Increase}

A major advantage of multipath is that it allows an increase of the overall throughput for a connection by bundling several links into one. This is somewhat similar to what BitTorrent does at the application layer by splitting data into chunks and transmitting them individually to different users. The difference is that it is significantly more efficient by being granular to the packet level and the fact that any application using the transport protocol in question can take advantage of it. One should also not condemn this on the basis of having similar questionable behaviour in regards to fairness as BitTorrent does [9].

The benefits as far as throughput is concerned are very much dependent on the application in question. In those cases where many small chunks of data must be transmitted the increase in speed will not be that noticeable. Video streaming however is an example in which the ability to bundle paths may enable a stream with significantly better quality to be transmitted in situations where this would not have been possible without a complex application level implementation due to link speed restrictions.

\subsection{Mobility}

A further major advantage of multipath is that it supports a form of mobility, which though slightly unconventional avoids many of the common pitfalls, such as triangular routing. In addition to this it supports a seamless handoff between connections, as it can simply use several connections concurrently. This last fact is of course dependent upon support from the underlying networking hardware.

Mobility is very simple in that it consists of adding and removing sockets as members of the logical socket. This means that as the device moves into range of a new access point it adds the received address to the connection and removes others when it leaves their range. In ideal cases this will lead to a situation where there is an overlap between networks, meaning there will be no handoff delay. With the right design this is not however a requirement, although a certain amount of thought must go into this part of the system as it has the potential to create a number of security holes such as connection hijacking, traffic redirection to be used for distributed denial of service attacks and eavesdropping at least part of the transmitted data. The last one is slightly questionable in the case of protocols including acknowledgements, as the eavesdropper would either have to 


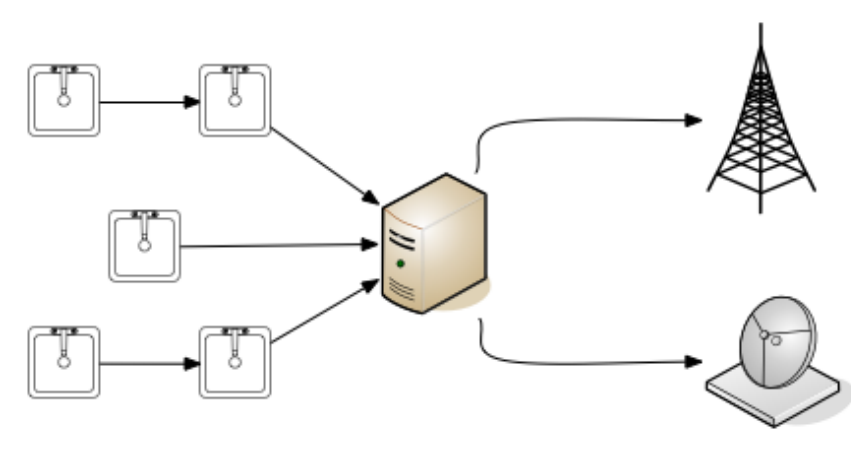

Figure 2: An ad-hoc wireless sensor network setup with a central sink using multipath backhaul.

not send them, in which case the link would be discarded as too lossy, or send acknowledgements which would cause data not to be delivered to the real destination host.

\section{SPECIFIC ISSUES}

\subsection{Scope}

\subsubsection{Gateway Functionality}

The main usage envisioned in a sensor network environment is for a system such as that shown in Figure 2. In this case the transport layer multipath would be employed on a gateway, which is most likely connected to the Internet or another network through different links, such as a satellite connection as well as a terrestrial radio technology to name a few examples.

There are a number of reasons not to employ multipath from the sensor nodes to the destination but the most significant are resource constraints. Establishing a number of connections and load-balancing between them is not significantly more resource intensive than a simple socket but with extremely resource constrained devices such as these it is still likely to be an issue. Problems could range from buffers growing slightly larger than they normally would due to variations in round trip time between the different links to the simple fact that initial implementations are unlikely to be as efficient as they could be. It is also fairly uncommon for such devices to have more than one form of network access.

In addition to this there is an additional per packet overhead, which depending on the implementation should not be significantly more than 16-bytes. This can be largely ignored when viewed in the light of standard network communications. In situations such as this where it is likely that a data link layer with a smaller MTU is used and a scheme such as 6lowpan [5][6] may be in operation this overhead is more significant and should be avoided if possible. Minimising the size and total number of packets is also very advantageous in busy networks, where even a small difference can significantly reduce collisions.

A further reason against employing transport layer multipath on the sensor nodes themselves is related to mobility. As described earlier mobility becomes fairly simple without the need for triangular routing. There is however a need for each connection to inform the destination of the newly received address. This means that by having a single link from a sink, rather than each node there is only the need to send a single notification of the address change, rather than one for every node. The result of this is a decrease in traffic on both the sensor network and the backhaul links. Furthermore it introduces the requirement for each node to have multiple addresses, which in the case of perhaps thousands of nodes could be seen as wasteful. This is however less of an issue if IPv6 is used.

\subsubsection{End-to-End Multipath}

As outlined above there are a lot of reasons for employing a transport layer multipath solution from a sink, rather than the nodes itself. This does not however mean that there are no cases in which a solution with multipath to the sensor level makes sense. In this case the sensor nodes in Figure 2 would simply use the sink as a router or bridge and two connections would be established from each node to the host system retrieving the data.

\subsection{Aggressiveness}

The chosen aggressiveness can have a major impact on the time taken to detect failures, at the cost of additional transmissions.

\subsection{Network Address Translation}

There are a number of issues with multipath, especially when employed with TCP, and network address translation. Whenever a NAT is only present on one side of the connection there is no problem, as that side can inform the other about any addresses it has. When there are however NATs at both ends neither of the hosts will be able to send addresses due the fact that they will not be aware of them.

As most of the concepts here apply to scenarios with IPv6 this should not be much of an issue however. In some cases one could imagine the sink itself acting as a NAT, in which case end-to-end multipath from the nodes would be an issue, but as outlined previously this is rarely advisable anyway.

\section{CONCLUSIONS}

The basic concept of transport layer multipath has been described. Several interesting uses have been outlined and justified, which all have potential to be further developed for more specific scenarios.

In addition to this a number of issues have been identified and possible solutions proposed for at least the major ones. Nothing has been found that would make such a system entirely unsuited for the use in sensor networks.

\section{ACKNOWLEDGEMENTS}

We would like to thank Mark Handley, who made us aware of the idea of multipath for TCP and through that triggered a lot of thought for applications of such a protocol. In addition to this we would like to thank the U-2010 project through which this work was financed.

\section{REFERENCES}

[1] M. Fiore and C. Casetti. An adaptive transport protocol for balanced multihoming of real-time traffic. In IEEE GLOBECOM 2005. IEEE Communications Society, 2005.

[2] D. Ganesan, R. Govindan, S. Shenker, and D. Estrin. Highly-resilient, energy-efficient multipath routing in wireless sensor networks. ACM SIGMOBILE Mobile 
Computing and Communications Review, 5(4):11 - 25, October 2001.

[3] J. Hunt, G. Nikolaidis, N. Stephan, and F. Vaisman. Multipath TCP: A Kernel-Level Implementation for Linux. Master's thesis, University College London, September 2008.

[4] J. R. Iyengar, P. Amer, and R. Stewart. Concurrent multipath transfer using sctp multihoming over independent end-to-end paths. In IEEE/ACM

Transactions on Networking 14, pages 951-964, 2006.

[5] N. Kushalnagar, G. Montenegro, and C. Schumacher. Rfc4919: Ipv6 over low-power wireless personal area networks (6lowpans). IETF: Network Working Group, August 2007.

[6] G. Montenegro, N. Kushalnagar, J. Hui, and D. Culler. Rfc4944: Transmission of ipv6 packets over ieee 802.15.4 networks. IETF: Network Working Group, September 2007.

[7] X. Qu, J. X. Yu, and R. P. Brent. A mobile tcp socket. Joint Computer Science Technical Report Series, April 1997.

[8] P. Savola. Ipv6 site multihoming using a host-based shim layer. In ICN/ICONS/MCL 2006, April 2006.

[9] D. Wischik, M. Handley, and M. B. Braun. The Resource Pooling Principle. ACM SIGCOMM Computer Communication Review, 38(5), October 2008. 